\title{
El aprendizaje a través de la simulación: Modelo de la UE en la asignatura "Instituciones Jurídicas de la Unión Europea"
}

\section{Learning through simulation: EU Model in the subject "Legal Institutions of the European Union"}

JOSÉ SORIANO GARCíA

ORCID: https://orcid.org/0000-0002-5739-7155

Departamento de Derecho Internacional

Público y Relaciones Internacionales

Universidad de Sevilla

jsoriano3@us.es

Fecha de recepción:

Fecha de aceptación:

DOI: http://dx.doi.org/10.12795/9788447221912.119

Pp.: 2631-2651 
El Ciclo de Mejora en el Aula (CIMA) que se presenta, se ha llevado a cabo en la asignatura Instituciones Jurídicas de la Unión Europea del Grado de Derecho. El objetivo del CIMA ha sido llevar a cabo una simulación del procedimiento legislativo ordinario de la Unión Europea (UE), para ello se ha situado al alumno en el centro del proceso, otorgándole a cada uno de ellos un rol dentro de las instituciones participantes. A través del desarrollo de este Modelo de la UE, el alumno ha construido el conocimiento a través de su experiencia, siendo parte activa de un procedimiento real de toma de decisiones, investigando el rol de su institución e interactuando de manera proactiva con el resto de sus compañeros presentes en las otras instituciones. Como resultado, la clase ha construido un acto legislativo de la UE a través de la encarnación de los diferentes actores mediante una simulación real del complejo sistema legislativo comunitario.

Palabras claves: Instituciones Jurídicas de la Unión Europea, Grado en Derecho, docencia universitaria, experimentación docente universitaria, procedimiento legislativo ordinario, codecisión.

\section{Abstract}

The Improvement Cycle in Classroom (ICIC) that is presented, has been carried out in the subject called Legal Institutions of the European Union of Law Degree. The aim of this ICIC is to develop a simulation of the ordinary legislative procedure of the European Union (EU). For this purpose, the students have been placed at the centre of the process, giving to each one a role within the participating institutions. Through the development of this EU Model, the students have built the knowledge through their own experience, being an active part of a real decision-making procedure, investigating the role of their institution and interacting proactively with the rest of their colleagues assigned to the other institutions. As a result of this proposal, the whole class has been able to build an EU legislative act through the incarnation of the different actors through a real simulation of the complex European legislative system.

Key Words: Legal Institutions of the European Union, Law Degree, University teaching, University teaching experimentation, Ordinary Legislative Procedure

Jornadas de Formación e Innovación Docente del Profesorado | № 2 (2019) 


\section{Breve Descripción del Contexto}

La asignatura en la que se ha implementado este CIMA es en Instituciones Jurídicas de la Unión Europea del Grado en Derecho. Se trata de un grupo numeroso en el que se encuentran matriculados 86 alumnos, asistiendo entre 70 y 80 a todas las sesiones. El grupo es poco diverso, tratándose mayoritariamente de alumnos que acaban de acceder a la universidad. La asignatura adolece de un problema fundamental al encontrarse ubicada en el primer cuatrimestre del primer curso. Al no haber cursado previamente Derecho Constitucional y Derecho Internacional Público, no conocen los fundamentos básicos de estos dos ordenamientos jurídicos, que se encuentran engarzados intrínsecamente con el ordenamiento jurídico europeo.

\section{Diseño previo del Ciclo de Mejora Docente}

\section{Mapa de Contenidos}

El mapa de contenidos reflejado en la Figura 1, tiene como objetivo relacionar contenidos que tradicionalmente se han estudiado de manera aislada: instituciones, procedimientos y actos legislativos, con el objetivo de lograr una visión de conjunto del funcionamiento real de las instituciones, a través de tres subproblemas: P1 - ¿Qué instituciones participan en el procedimiento legislativo europeo?; P2 - ¿Cómo es el procedimiento que se lleva a cabo?; P3 ¿Qué tipo de actos elabora la Unión Europea? A través de estos tres problemas interrelacionados se intenta lograr una visión completa de un procedimiento que tradicionalmente ha sido estudiado a través de compartimentos estancos.

Jornadas de Formación e Innovación Docente del Profesorado | № 2 (2019)

Esta obra se distribuye con la licencia Creative Commons 


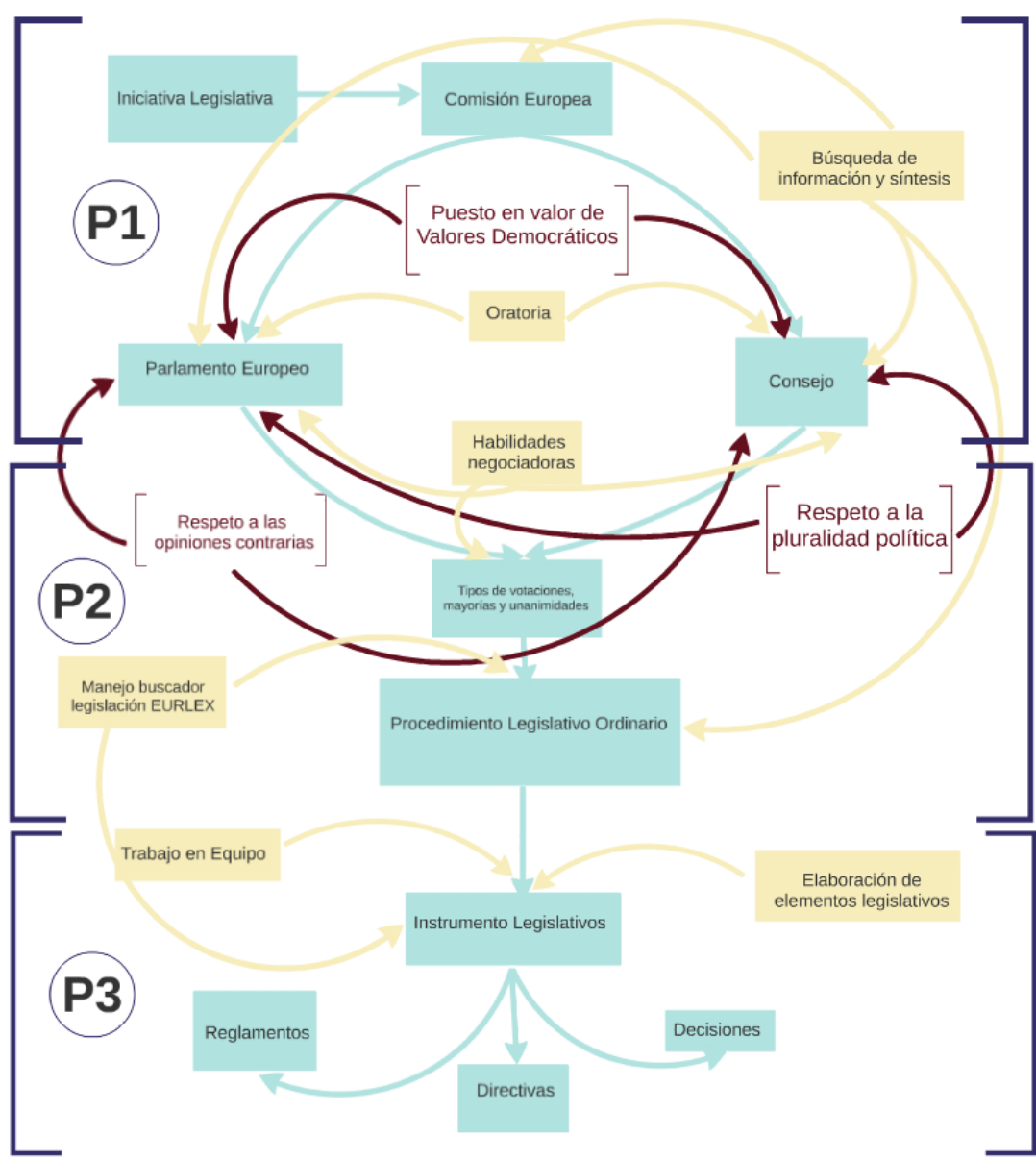

Figura 1. Mapa de Contenidos

\section{Modelo Metodológico}

La Metodología que se ha llevado a cabo ha cambiado el paradigma de la tradicional clase magistral, ubicando al alumno en el centro del proceso de aprendizaje, siendo el actor clave del mismo a través de una simulación real del complejo procedimiento legislativo de la UE.

Jornadas de Formación e Innovación Docente del Profesorado | № 2 (2019)

Esta obra se distribuye con la licencia Creative Commons Reconocimiento-NoComercial-SinObraDerivada Internacional (CC BY-NC-ND 4.0.) 


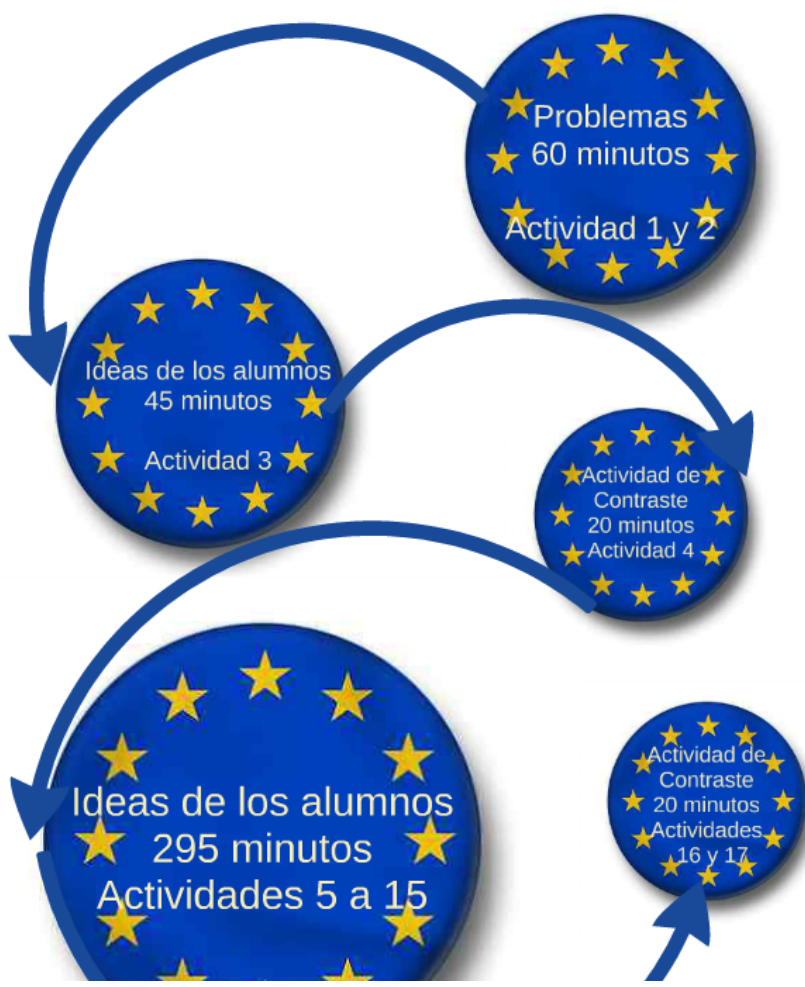

Figura 2. Modelo metodológico implementado.

Jornadas de Formación e Innovación Docente del Profesorado | № 2 (2019) 


\section{Tabla 1. Secuencia de actividades del ciclo de mejora en el aula.}

\begin{tabular}{|c|c|c|}
\hline \multicolumn{3}{|c|}{ Sesión 1 (Miércoles 23/10 - 1h) } \\
\hline Actividad & Descripción & Tiempo \\
\hline $\begin{array}{c}\text { № } 1 \\
\text { Vídeo - } \\
\text { Presentación } \\
\text { de los } \\
\text { problemas }\end{array}$ & $\begin{array}{l}\text { Visionaremos el vídeo de VisualPolitik “¿Es la Unión } \\
\text { Europea realmente democrática? (8:40). } \\
\text { En esta actividad se introducen de manera clara las } \\
\text { diferentes instituciones de la Unión Europea y sirve como } \\
\text { primera toma de contacto con los diferentes problemas } \\
\text { que se quieren ver en el CIMA. } \\
\text { ¿Qué instituciones participan en el procedimiento } \\
\text { legislativo europeo? } \\
\text { ¿Cómo es el procedimiento que se lleva a cabo? } \\
\text { ¿Qué tipo de actos legislativos elabora la Unión } \\
\text { Europea? } \\
\text { A lo largo del vídeo lo pararemos para introducir } \\
\text { algunos aspectos que considero fundamentales y que } \\
\text { deben ser concretados. }\end{array}$ & $25 \mathrm{~min}$ \\
\hline $\begin{array}{c}\text { № } 2 \\
\text { Organización } \\
\text { de la actividad }\end{array}$ & $\begin{array}{l}\text { Esta actividad es la piedra angular del CIMA, ya que } \\
\text { estructurará el trabajo que deberán llevar a cabo los } \\
\text { alumnos en el resto de sesiones, así como el trabajo que } \\
\text { el alumnado tendrá que realizar de manera autónoma. } \\
\text { Previamente en la Enseñanza Virtual colgaré la } \\
\text { distribución de los alumnos en los diferentes roles que } \\
\text { tendrán que ejecutar. El día del cuestionario inicial, se } \\
\text { dio tiempo a los alumnos de tiempo para que se apunten } \\
\text { a la actividad. Atendiendo al número de alumnos } \\
\text { apuntados se les dividirá en las siguientes instituciones } \\
\text { - Consejo - divididos por países. } \\
\text { - Parlamento Europeo - dividido por grupos políticos. } \\
\text { - Comisión Europea.. } \\
\text { Se utilizará un pequeño vídeo de } 3-4 \text { minutos para que } \\
\text { visualicen a las personas fisicas cuyo rol asumirán. } \\
\text { Se les explicará la tarea que debe llevar a cabo cada } \\
\text { uno de los grupos (Nota informativa } 1 \text { que se dejará en } \\
\text { la Enseñanza Virtual). }\end{array}$ & $35 \mathrm{~min}$ \\
\hline
\end{tabular}

Jornadas de Formación e Innovación Docente del Profesorado | № 2 (2019)

Esta obra se distribuye con la licencia Creative Commons Reconocimiento-NoComercial-SinObraDerivada $\quad 4.0$ Internacional (CC BY-NC-ND 4.0.) 


\begin{tabular}{|c|c|c|}
\hline & $\begin{array}{l}\text { Se solicitará que una pareja dentro de cada grupo } \\
\text { realice en la siguiente sesión (lunes, disponen de } \\
\text { cuatro días y medio) una presentación al resto de } \\
\text { sus compañeros sobre la institución (composición, } \\
\text { objetivos y funciones, destacando fundamental- } \\
\text { mente su rol en el proceso legislativo). } \\
\text { - Se entregará una propuesta de Directiva en materia } \\
\text { de medio ambiente, y se les solicitará a los miem- } \\
\text { bros del Consejo y de los diferentes grupos políticos } \\
\text { del Parlamento Europeo, que lleven a cabo un do- } \\
\text { cumento de posición sobre dicha directiva, explici- } \\
\text { tando sus líneas rojas, así como los ámbitos en que } \\
\text { están más dispuesto a ceder y negociar. } \\
\text { Con objeto de facilitar su labor, les facilitaré materiales } \\
\text { y web de referencia. }\end{array}$ & \\
\hline \multicolumn{3}{|c|}{ Sesión 2 (Lunes 28/10 - 2h) } \\
\hline Actividad & Descripción & Tiempo \\
\hline $\begin{array}{c}\text { №3 } \\
\text { Exposición de } \\
\text { los alumnos }\end{array}$ & $\begin{array}{l}\text { Los alumnos llevarán a cabo la presentación de las tres } \\
\text { instituciones. Al finalizar la exposición de cada uno, mi } \\
\text { labor consistirá en formular preguntas sobre aquellos } \\
\text { aspectos que no hayan quedado claros, así como hacer } \\
\text { una síntesis con los elementos que vayan a sernos de } \\
\text { utilidad para la simulación. }\end{array}$ & $45 \mathrm{~min}$ \\
\hline $\begin{array}{l}\text { Nou } \\
\text { Actividad de } \\
\text { Contraste }\end{array}$ & $\begin{array}{l}\text { Utilizando los inputs de las exposiciones, me apoyaré en } \\
\text { una infografia del proceso legislativo ordinario con objeto } \\
\text { de completar y debatir en clase de manera conjunta, los } \\
\text { pasos de los que se compone el procedimiento. }\end{array}$ & $20 \mathrm{~min}$ \\
\hline $\begin{array}{l}\text { №5 } \\
\text { Trabajo en } \\
\text { clase }\end{array}$ & $\begin{array}{l}\text { La segunda hora de clase, la clase se dividirá en dos, lo } \\
\text { que están adscritos al Consejo y al Parlamento Europeo. } \\
\text { El objetivo es que empiecen a negociar entre ellos y } \\
\text { conozcan las posiciones del resto de grupos. Durante } \\
\text { este tiempo mi rol será el de pasearme por los diferentes } \\
\text { grupos, aconsejando en caso de que sea necesario, y } \\
\text { aclarando las reglas de procedimiento que se tendrán } \\
\text { que seguir con objeto de que la simulación se pueda } \\
\text { llevar de manera fluida. }\end{array}$ & 55 min \\
\hline \multicolumn{3}{|c|}{ Sesión 3 (Martes 29/10 - 1h) } \\
\hline Actividad & Descripción & Tiempo \\
\hline $\begin{array}{c}\text { №6 } \\
\text { Simulación }\end{array}$ & $\begin{array}{l}\text { En este ejercicio, se llevará a cabo la primera lectura del } \\
\text { Parlamento Europeo. Cada grupo político intervendrá } \\
\text { exponiendo su posición política. Mi rol, en tanto que } \\
\text { presidente de la eurocámara, será la de moderar el } \\
\text { debate y evitar las interrupciones, fomentaré eso sí, las } \\
\text { interpelaciones. }\end{array}$ & $40 \mathrm{~min}$ \\
\hline
\end{tabular}

Jornadas de Formación e Innovación Docente del Profesorado | № 2 (2019)

Esta obra se distribuye con la licencia Creative Commons Reconocimiento-NoComercial-SinObraDerivada $\quad 4.0$ Internacional (CC BY-NC-ND 4.0.) 


\begin{tabular}{|c|c|c|}
\hline $\begin{array}{l}\text { No97 } \\
\text { Elaboración de } \\
\text { enmiendas }\end{array}$ & $\begin{array}{l}\text { Al levantarse la sesión, los grupos tendrán tiempo para } \\
\text { debatir las enmiendas y lograr apoyos. Dispondrán hasta } \\
\text { la próxima sesión para presentar un documento apoyado } \\
\text { por los diferentes grupos parlamentarios. Les orientaré } \\
\text { en la redacción de las enmiendas en caso de que se vean } \\
\text { atascados. }\end{array}$ & $20 \mathrm{~min}$ \\
\hline \multicolumn{3}{|c|}{ Sesión 4 (Miércoles 30/10 - 1h) } \\
\hline $\begin{array}{c}\text { No8 } \\
\text { Votación }\end{array}$ & $\begin{array}{l}\text { Al principio de la sesión se procederán a votar las } \\
\text { diferentes enmiendas que hayan producido los diferentes } \\
\text { grupos parlamentarios del Parlamento Europeo. }\end{array}$ & $10 \mathrm{~min}$ \\
\hline $\begin{array}{l}\text { No9 } \\
\text { Simulación }\end{array}$ & $\begin{array}{l}\text { Se llevará a cabo la primera lectura del Consejo en su } \\
\text { formación de medio ambiente. El procedimiento será } \\
\text { similar al de la actividad } 7 \text {. En tanto que Presidente del } \\
\text { Consejo, mi función será la de orientar los posibles } \\
\text { puntos de acuerdo, así como recordarles que la votación } \\
\text { será sobre las enmiendas del Parlamento Europeo que } \\
\text { les iré desgranando. }\end{array}$ & $40 \mathrm{~min}$ \\
\hline $\begin{array}{c}\text { №10 } \\
\text { Votación }\end{array}$ & $\begin{array}{l}\text { Se llevará a cabo una votación sobre las enmiendas del } \\
\text { Parlamento Europeo. En caso de que no sean adoptadas } \\
\text { absolutamente todas. Se instará a los miembros del } \\
\text { Consejo a que presenten enmiendas adicionales a las } \\
\text { enmiendas del Parlamento Europeo aprobados por ellos. } \\
\text { Los Estados tendrán hasta el sábado para presentar } \\
\text { enmiendas, las enmiendas deberán estar apoyadas por } \\
\text { un mínimo de cinco estados para poder ser votadas. }\end{array}$ & $10 \mathrm{~min}$ \\
\hline \multicolumn{3}{|c|}{ Sesión 5 (Lunes4/11 - 2h) } \\
\hline $\begin{array}{c}\text { No11 } \\
\text { Votación }\end{array}$ & $\begin{array}{l}\text { Al principio de la sesión se procederán a votar las } \\
\text { diferentes enmiendas que hayan producido los } \\
\text { diferentes estados del Consejo. }\end{array}$ & $20 \mathrm{~min}$ \\
\hline $\begin{array}{l}\text { No12 } \\
\text { Simulación }\end{array}$ & $\begin{array}{l}\text { El Parlamento Europeo votará en bloque el documento } \\
\text { producido por el Consejo en esta segunda lectura. En } \\
\text { caso de que no se adopte en su totalidad se llevará a cabo } \\
\text { una discusión para la inclusión de nuevas enmiendas. }\end{array}$ & $20 \mathrm{~min}$ \\
\hline $\begin{array}{l}\text { No13 } \\
\text { Elaboración } \\
\text { y votación de } \\
\text { enmiendas }\end{array}$ & $\begin{array}{l}\text { Los grupos políticos deberán llevar a cabo la elaboración } \\
\text { de nuevas enmiendas que se unirán a las que ya se han } \\
\text { ido aprobando. } \\
\text { Cuando hayan elaborado las enmiendas se procederá a } \\
\text { su votación. }\end{array}$ & $40 \mathrm{~min}$ \\
\hline $\begin{array}{c}\text { № } 14 \\
\text { Votación }\end{array}$ & $\begin{array}{l}\text { Los representantes de la Comisión se pronunciarán } \\
\text { positivamente o negativamente sobre las Enmiendas }\end{array}$ & $5 \mathrm{~min}$ \\
\hline
\end{tabular}

Jornadas de Formación e Innovación Docente del Profesorado | № 2 (2019)

Esta obra se distribuye con la licencia Creative Commons Reconocimiento-NoComercial-SinObraDerivada $\quad 4.0$ Internacional (CC BY-NC-ND 4.0.) 


\begin{tabular}{|c|c|c|}
\hline $\begin{array}{c}\text { № } 15 \\
\text { Debate y } \\
\text { Votación }\end{array}$ & $\begin{array}{l}\text { Los representantes del Consejo votarán sobre las } \\
\text { Enmiendas del Parlamento Europeo de manera } \\
\text { diferenciada según el posicionamiento de la Comisión. } \\
\text { Antes de la votación de cada una de las enmiendas, } \\
\text { los diferentes estados podrán intervenir con objeto de } \\
\text { convencer sobre el sentido de su voto al resto de los } \\
\text { estados. }\end{array}$ & $35 \mathrm{~min}$ \\
\hline \multicolumn{3}{|c|}{ Sesión 6 (Martes 4/11 - 1h) } \\
\hline $\begin{array}{c}\text { № } 16 \\
\text { Simulación } \\
\text { Comité de } \\
\text { Conciliación }\end{array}$ & $\begin{array}{l}\text { En esta opción se llevará a cabo una sesión conjunta } \\
\text { Consejo-Parlamento Europeo, en la que se intentará en } \\
\text { una discusión lograr una solución aceptable para las dos } \\
\text { partes. El documento que salga, se someterá a votación } \\
\text { del Parlamento Europeo y del Consejo. }\end{array}$ & $30 \mathrm{~min}$ \\
\hline № 17 & Contestación del mismo cuestionario inicial. & $30 \mathrm{~min}$ \\
\hline $\begin{array}{c}\text { № } 18 \\
\text { Sintesis }\end{array}$ & $\begin{array}{l}\text { Con objeto de asentar los conocimientos, se llevará a } \\
\text { cabo una actividad final en la que se repasarán todos } \\
\text { los conceptos vistos a lo largo de la actividad. En esta } \\
\text { actividad se articulará un diálogo socrático en la que los } \\
\text { alumnos vayan contestando, encargándome de moderar } \\
\text { y matizar ciertos puntos. Esta actividad se llevará a cabo } \\
\text { en la siguiente sesión, posterior a las } 8 \text { h del CIMA } 2 \text {. }\end{array}$ & \\
\hline
\end{tabular}

\section{Cuestionario Inicial}

Con objeto de analizar de conocer las ideas previas del alumnado, se pasó un formulario de tres preguntas, que se corresponden con los tres problemas señalados en el modelo metodológico. Atendiendo a los resultados obtenidos podremos realizar modificaciones en la secuencia de actividades y al mismo tiempo nos servirá como elemento fundamental para analizar la evolución del aprendizaje del alumnado.

Buenos días Sra. Von Der Leyen,

Antes que nada, ¡Enhorabuena por su reciente elección como Presidenta de la Comisión Europea!, es usted la primera mujer en ostentar este cargo y estar al frente de más de 500 millones de europeos.

Soy Martine Lefebvre, funcionaria de la Comisión Europea y la persona encargada de ayudarle en sus primeros dias en Bruselas. Ya me ha

Jornadas de Formación e Innovación Docente del Profesorado | № 2 (2019)

Esta obra se distribuye con la licencia Creative Commons

Reconocimiento-NoComercial-SinObraDerivada $\quad 4.0$ Internacional (CC BY-NC-ND 4.0.) 
comunicado su jefa de gabinete que quiere empezar a trabajar rápidamente y que su primera medida va a ser en relación con el medio ambiente, con lo que no perdamos ni un solo minuto y manos a la obra.

Con objeto de serle de la mayor utilidad posible y poner en marcha un proceso formativo acorde a sus necesidades, necesitaría que me contestase a una serie de preguntas, ya que como verá, el procedimiento legislativo alemán no tiene nada que ver con el europeo.

1) ¿Qué instituciones participan en el proceso legislativo europeo?

2) ¿Cómo es el proceso que tiene lugar desde que elegimos una problemática, medio ambiente que es lo que nos atañe urgentemente, hasta que empiezan a verse resultados en la vida de los ciudadanos?

3) ¿Qué tipo de actos legislativos elabora la Unión Europea? ¿Sabe usted la diferencia entre ellos?

\section{Aplicación del Ciclo de Mejora Docente}

\section{Descripción de las sesiones}

\section{Sesión 0}

Debido a las necesidades logísticas de este CIMA 2, en la sesión que tuvo lugar dos días antes de la sesión 1, expliqué brevemente que el miércoles iba a comenzar una actividad diferente, consistente en una simulación del procedimiento legislativo ordinario de la Unión Europea. Les solicité que, para la clase siguiente, aquellas personas que quisiesen participar y que se comprometieran a realizar las tareas que se les encomendase, deberían apuntarse en una lista que me darían en la siguiente sesión. Recogí la lista, en la que se apuntaron 75 alumnos, el $84 \%$ de los alumnos matriculados en el curso. En esta misma sesión pasé el cuestionario inicial.

\section{Sesión 1 (Miércoles 23/10 - 1h).}

\section{Actividad 1}

En esta primera actividad visualizamos el vídeo de Visual Politik, ¿Es la Unión Europea, realmente democrática?

Jornadas de Formación e Innovación Docente del Profesorado | № 2 (2019)

Esta obra se distribuye con la licencia Creative Commons Reconocimiento-NoComercial-SinObraDerivada $\quad 4.0$ Internacional (CC BY-NC-ND 4.0.) 
La actividad salió tal y como estaba prevista. El vídeo suscitó cierto interés al alumnado, al igual que un poco de desazón, ya que pudieron observar lo complejo que es el procedimiento legislativo de la Unión Europea. Tras esta presentación de la problemática, ciertamente compleja, me permitió introducir de manera favorable la estrategia de simulación, ya que justifiqué la dificultad del mecanismo con la necesidad de utilizar un nuevo enfoque metodológico que les facilitase la tarea. Esta idea fue mayoritariamente aceptada.

\section{Actividad 2}

En esta actividad, consistente en la organización de todo el sistema, lo primero que hice fue leer los componentes de cada país, de cada grupo político y de la Comisión Europea, con objeto de facilitar la identificación entre ellos, ya que, al ser un grupo tan numeroso, muchos de ellos no se conocen. En segundo lugar, leí la propuesta de Directiva de medioambiente sobre la que tendrán que trabajar y posteriormente expliqué pormenorizadamente las tareas que tendrían que llevar a cabo cada uno de ellos, dependiendo del papel que les hubiese tocado. Debido a las numerosas preguntas y dudas expliqué varias veces qué es lo que tenía que hacer exactamente cada uno.

Al salir de clase, lo primero que hice fue plasmar por escrito en la Enseñanza Virtual todas las indicaciones que les había dado. Con objeto de facilitarles la tarea para llevar a cabo los documentos de posición, aparte de las webs de referencia institucionales que vimos en clase, les dejé diferentes materiales que les sirviesen de ayuda. Disponían desde el miércoles hasta el lunes para enviar los documentos de posición. Todos los grupos políticos, excepto uno, enviaron su documento, así como 25 de los 28 países.

\section{Sesión2 (Lunes 28/10 - 2h).}

Al comenzar la sesión fui abordado por aquellos alumnos que no habían enviado su documento de posición, así

Jornadas de Formación e Innovación Docente del Profesorado | № 2 (2019) 
como por alumnos que tenían dudas relativas al procedimiento. Con objeto de solventar las dudas, dediqué $10 \mathrm{mi}-$ nutos al comienzo de la sesión a volver a aclarar dudas sobre el procedimiento.

\section{Actividad 3 y Actividad 4}

Los tres grupos llevaron a cabo las presentaciones orales, con resultados muy dispares entre los mismos.

1. La presentación de la Comisión Europea fue excelente. Se ciñeron a las instrucciones de manera clara y concisa y explicaron los conceptos fundamentales con rigor. Al finalizar su exposición les hice algunas preguntas que respondieron sin problemas, y posteriormente llevé a cabo una síntesis de los puntos principales e indiqué el rol que iban a tener a lo largo de la simulación.

2. A diferencia de la presentación de la Comisión Europea, la presentación del Consejo la llevaron a cabo cuatro personas y colaboraron ocho en la elaboración de la presentación. En esta ella los alumnos se equivocaron en los conceptos fundamentales, viéndome forzado a parar en ciertos momentos la intervención para clarificar algunos puntos, con objeto de que no se confundiesen sus compañeros. Al finalizar remarqué los puntos positivos de la presentación, y llevé a cabo una pequeña síntesis de estas instituciones e indiqué el rol que tendría el Consejo a lo largo de la simulación.

3. La presentación del Parlamento Europeo, fue correcta desde un punto de vista de explicación de los conceptos, pero adolecía de varios problemas, no fue absolutamente nada visual y fue excesivamente densa, copiando artículo tras artículo, no transmitiendo de manera clara los conceptos fundamentales y explicando pormenorizadamente puntos de poca relevancia. Al igual que en las otras dos presentaciones me centré en los puntos principales de la presentación a modo de sintesis y expliqué

Jornadas de Formación e Innovación Docente del Profesorado | № 2 (2019) 

simulación.

Al finalizar la sesión les dejé las diapositivas con las que suelo trabajar en las sesiones tradicionales, les dije que se las leyesen a modo de repaso y fundamentalmente para que les sirviese de cara al examen final de febrero. También les proporcioné la infografía del procedimiento legislativo ordinario, pero me centré solo en el principio del procedimiento, la primera lectura, ya que de ponerlo entero podría agobiarlos debido a la dificultad del proceso, no subiéndolo por tanto a la Enseñanza Virtual.

\section{Actividad 5}

En esta actividad, que en principio debería durar 55 minutos, empezó más tarde debido al retraso en las exposiciones. Por lo tanto, esta actividad empezó tras $1 \mathrm{~h} 30$ de clase, con el correspondiente cansancio, tras llevar los alumnos desde las 8 h30 de clases ininterrumpidas y ser las $14 \mathrm{~h}$ al comenzar la actividad. Durante los primeros 15 minutos los alumnos se pusieron a trabajar entre ellos, tuve que animarles a que se levantaran y buscaran al resto de grupos parlamentos y países. Durante este tiempo se trabajó de manera adecuada, muchos incluso empezaron a escribir posibles enmiendas. Durante este tiempo fui pasando por los diferentes grupitos solucionando dudas que me planteaban y dando algún ejemplo de enmienda. Sin embargo, a los 15 minutos, una cantidad relevante, fundamentalmente los representantes de países menos activos, empezaron a recoger, ante tal situación, permití que quien quisiera irse podía hacerlo, pero que era recomendable que siguiesen negociando. Solo la mitad se quedó debatiendo.

\section{Sesión 3 (Martes 29/10 - 1h)}

\section{Actividad 6}

En esta actividad tuvo lugar el primer debate, al llegar a clase ubiqué a los representantes del Parlamento al principio de la clase y a los del Consejo detrás, todos con sus

Jornadas de Formación e Innovación Docente del Profesorado | № 2 (2019) 
correspondientes carteles de su país o su grupo político. En mi rol en tanto que Presidente de la Cámara otorgué la palabra para una primera posición inicial de cada grupo político, para a continuación comenzar con las interpelaciones. En general el debate fue animado, con numerosas réplicas y contrarréplicas, los alumnos, en diverso grado se metieron en el papel y el debate fue muy fructífero. Durante toda la sesión tuve de fondo de pantalla la composición del Parlamento Europeo. Con respecto a la parte de la clase que no intervino en la actividad (los Estados), algunos se entusiasmaron con el debate parlamentario mientras que otros desconectaron.

\section{Actividad 7}

Esta actividad no se llegó a celebrar debido a que el debate ocupó la hora entera. Creo que fue positivo que no tuviese lugar, ya que en el mismo debate los representantes políticos empezaron a buscar posibles enmiendas conjuntas. Al finalizar la clase llevé a cabo una Junta de Portavoces en que les indiqué la manera en que tendrían que presentar las enmiendas para ser votadas al día siguiente.

\section{Sesión 4 (Miércoles 30/10 - 1h)}

\section{Actividad 8}

Antes de comenzar la clase recibí 36 enmiendas por parte de los diferentes grupos parlamentarios. Muchas de las enmiendas fueron presentadas por varios grupos parlamentarios, disponiendo ya de una mayoría previa antes de la votación. A continuación, se procedió a la votación de las enmiendas. Para ello se utilizó la siguiente calculadora de votaciones: https://resultados-elecciones.eu/ herramientas/calculadora-de-mayorias/.En total se aprobaron 22 de las 36 enmiendas presentadas.

\section{Actividad 9}

A continuación, tuvo lugar la sesión plenaria del Consejo. Aunque un debate con 28 grupos parte de una

Jornadas de Formación e Innovación Docente del Profesorado | № 2 (2019) 
dificultad mayor, el debate fue animado, los alumnos que disponían de países con una gran población empujaron el debate y empezaron a verse principios de acuerdo entre ellos.

\section{Actividad 10}

Debido a que el debate de la actividad 9 llegó hasta el final de la clase, y también con objeto de que pudiesen leer las enmiendas aprobadas por el Parlamento, indiqué que el voto sería telemático, y que me debería enviar cada país sus votaciones a mí directamente. También les indiqué la manera en que me tenían que enviar las enmiendas para que fuesen votadas en la sesión del lunes. Con objeto de que conociesen el sistema de votaciones les dejé la calculadora de votaciones del Consejo: https:// www.consilium.europa.eu/es/council-eu/voting-system/ voting-calculator/

Cuando recibí las enmiendas, llevé a cabo las votaciones y subí los resultados a la Enseñanza Virtual, volviendo a orientarles sobre la presentación de las enmiendas. El domingo recibí una gran cantidad de correos electrónicos en el que cada país me enviaba sus enmiendas con sus apoyos y al mismo tiempo se coordinaron para presentarlas todas en un único documento. Posteriormente les dejé otra nota informativa indicado el procedimiento que tendría lugar al día siguiente. Con objeto de introducir los órganos consultivos, les dejé tres enmiendas elaboradas por mí que se votarían también en la siguiente sesión.

\section{Sesión 5 (Lunes 4/11 - 2h)}

\section{Actividad 11 y Actividad 12}

Al comenzar la sesión volví a explicar la dinámica de la votación, así como la manera en que debían proceder con las enmiendas presentadas por los órganos consultivos. Justo a continuación se llevó a cabo la votación de las 122 enmiendas. Debido a la gran cantidad de enmiendas

Jornadas de Formación e Innovación Docente del Profesorado | № 2 (2019)

Esta obra se distribuye con la licencia Creative Commons 
y ayudándome de la representante de Finlandia (ejerce la presidencia pro-témpore del Consejo) fuimos votando todas las enmiendas, no votando enmiendas similares ya aprobadas, con objeto de que la votación fuese más ágil y el documento final más coherente. A lo largo de la sesión, la Comisión Europea llevó a cabo precisiones jurídicas sobre si la votación debía ser por mayoría cualificada o por unanimidad. A lo largo de la votación, los miembros del Parlamento fueron trabajando de manera conjunta con objeto de definir su sentido del voto. Al finalizar la votación, volví sobre la infografía del procedimiento legislativo, explicando el punto en que nos encontrábamos.

El Parlamento Europeo votó la posición del Consejo, decidiendo continuar con el procedimiento legislativo, pero incluyendo nuevas enmiendas. A continuación, votaron solamente sobre las enmiendas recién aprobadas por el Consejo. Debido a que se habían consumido casi las dos horas de clase, la actividad 13, 14, 15 y 16 tuvieron lugar en la siguiente sesión. Para finalizar, les indiqué a los miembros del Parlamento en segunda lectura que las enmiendas debían elaborarlas en casa para que solo fuesen votadas en clase.

\section{Sesión 6 (Martes 5/11 - 2h).}

\section{Actividad 13 y Actividad 14.}

Al comenzar la sesión, tuvo lugar una breve defensa de las enmiendas presentadas y se votaron las ocho enmiendas por el Parlamento Europeo, aprobándose cinco de las ocho. Con objeto de que la Comisión pudiese pronunciarse a favor de las mismas y votar por tanto el Consejo por mayoría cualificada. La Comisión negoció la reformulación de dos enmiendas con los grupos políticos, lográndose un acuerdo.

Jornadas de Formación e Innovación Docente del Profesorado | № 2 (2019) 
Actividad 15.

A continuación tuvo lugar una sesión del Consejo, debatiéndose en exclusiva sobre las enmiendas aprobadas por el Parlamento Europeo. Al finalizar se produjo la votación, aprobándose cuatro de las cinco enmiendas por parte del Consejo.

\section{Actividad 16}

Se llevó a cabo un Comité de Conciliación, dándoles cinco minutos para que negociara directamente el Parlamento con el Consejo sobre la Enmienda no aprobada. Tras lograr un acuerdo, la enmienda se sometió a votación, no siendo aprobada. Tras ello, se procedió a la votación del documento final, con todas las enmiendas aprobadas por ambas Cámaras en las diferentes lecturas. La Comisión se encargó de elaborar el documento final. Finalmente, la Directiva con 73 artículos fue aprobada con una amplia mayoría tanto en el Consejo como en el Parlamento Europeo.

La contestación al cuestionario final tuvo lugar en la siguiente sesión, ya fuera del horario del Ciclo de Mejora en el Aula, aunque sus resultados se incluyen en este trabajo.

\section{Evaluación del aprendizaje de los estudiantes}

Tal y como se explicó en el diario de sesiones, al finalizar la simulación se volvió a pasar el cuestionario inicial (ahora final), extrayendo las escaleras de aprendizaje, así como un análisis comparativo de las respuestas individualizadas de los alumnos. En las escaleras se agruparon las respuestas del alumnado en diferentes niveles (A, B, C, D, $E)$, reflejando la altitud de los escalones, la dificultad para pasar de un nivel a otro. El resultado ha sido satisfactorio, ya que la evolución del aprendizaje de la inmensa mayoría del alumnado ha sido notable.

Jornadas de Formación e Innovación Docente del Profesorado | № 2 (2019)

Esta obra se distribuye con la licencia Creative Commons 


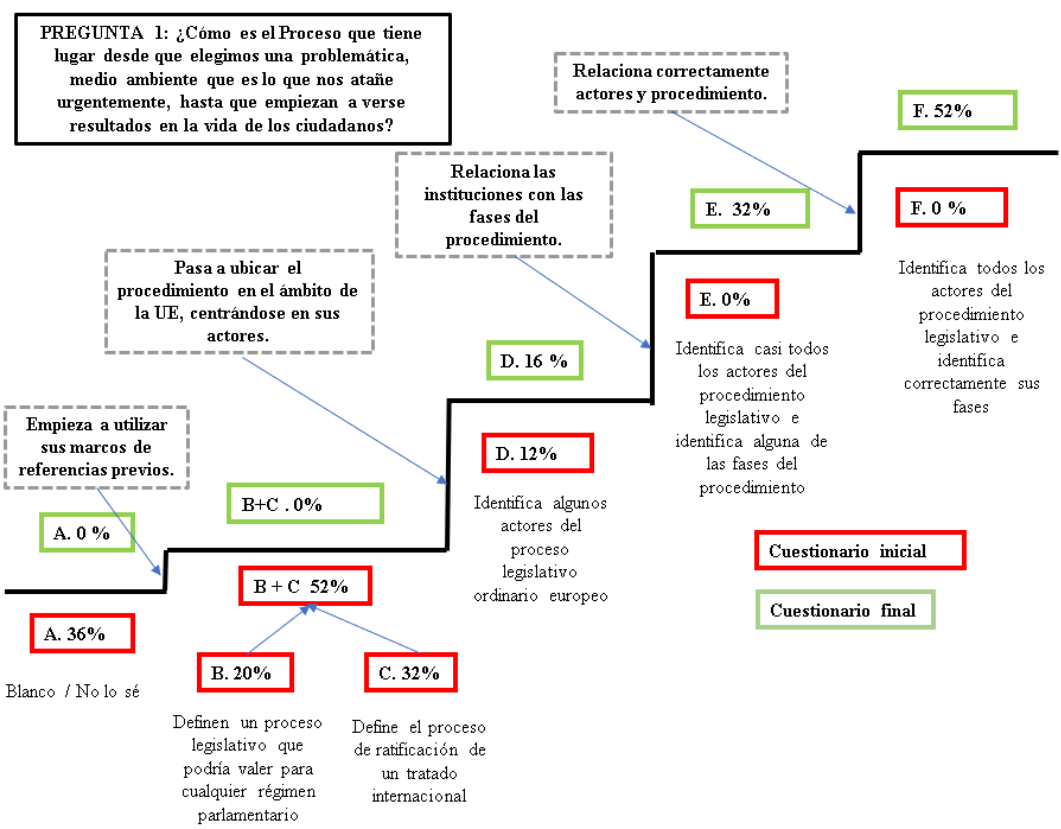

Figura 3. Escalera 1

PREGUNTA 2: ¿Qué tipos de actos legislativos elabora la Unión Europea? ¿Sabe usted la diferencia entre ellos?

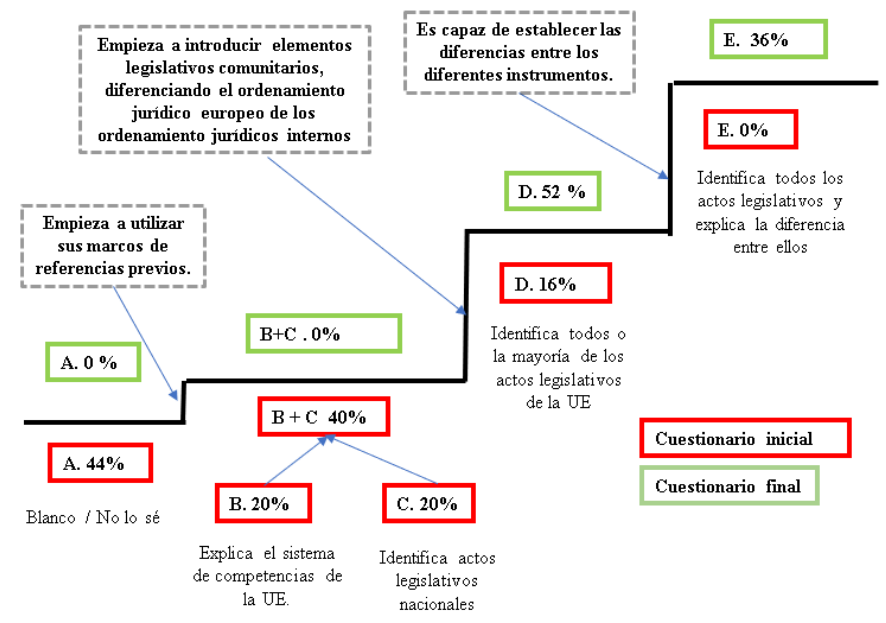

Figura 4. Escalera 2

Jornadas de Formación e Innovación Docente del Profesorado | № 2 (2019) 
Tabla 2. Análisis comparativo de cuestionarios iniciales y finales.

\begin{tabular}{|c|c|c|c|c|c|c|c|c|c|c|c|c|c|}
\hline \multicolumn{10}{|c|}{ Análisis comparativo cuestionarios iniciales y finales } \\
\hline & \multicolumn{1}{|c|}{ Pregunta 1 } & \multicolumn{2}{c|}{ Pregunta 2 } & \multicolumn{3}{c|}{ Pregunta 1 } & \multicolumn{3}{c|}{ Pregunta 2 } \\
\hline Sujeto 1 & $\mathrm{B} / \mathrm{C}$ & $\mathrm{E}$ & $\uparrow \uparrow$ & $\mathrm{B} / \mathrm{C}$ & $\mathrm{E}$ & $\uparrow \uparrow$ & Sujeto 14 & $\mathrm{A}$ & $\mathrm{F}$ & $\uparrow \uparrow \uparrow \uparrow$ & $\mathrm{D}$ & $\mathrm{E}$ & $\uparrow$ \\
\hline Sujeto 2 & $\mathrm{D}$ & $\mathrm{F}$ & $\uparrow \uparrow$ & $\mathrm{A}$ & $\mathrm{E}$ & $\uparrow \uparrow \uparrow$ & Sujeto 15 & $\mathrm{A}$ & $\mathrm{F}$ & $\uparrow \uparrow \uparrow \uparrow$ & $\mathrm{A}$ & $\mathrm{D}$ & $\uparrow \uparrow$ \\
\hline Sujeto 3 & $\mathrm{B} / \mathrm{C}$ & $\mathrm{E}$ & $\uparrow \uparrow$ & $\mathrm{A}$ & $\mathrm{E}$ & $\uparrow \uparrow \uparrow$ & Sujeto 16 & $\mathrm{B} / \mathrm{C}$ & $\mathrm{E}$ & $\uparrow \uparrow$ & $\mathrm{A}$ & $\mathrm{D}$ & $\uparrow \uparrow$ \\
\hline Sujeto 4 & $\mathrm{D}$ & $\mathrm{E}$ & $\uparrow$ & $\mathrm{A}$ & $\mathrm{D}$ & $\uparrow \uparrow$ & Sujeto 17 & $\mathrm{B} / \mathrm{C}$ & $\mathrm{F}$ & $\uparrow \uparrow \uparrow$ & $\mathrm{A}$ & $\mathrm{B}$ & $\uparrow$ \\
\hline Sujeto 5 & $\mathrm{A}$ & $\mathrm{F}$ & $\uparrow \uparrow \uparrow \uparrow$ & $\mathrm{B} / \mathrm{C}$ & $\mathrm{D}$ & $\uparrow$ & Sujeto 18 & $\mathrm{A}$ & $\mathrm{F}$ & $\uparrow \uparrow \uparrow \uparrow$ & $\mathrm{B} / \mathrm{C}$ & $\mathrm{D}$ & $\uparrow$ \\
\hline Sujeto 6 & $\mathrm{B} / \mathrm{C}$ & $\mathrm{F}$ & $\uparrow \uparrow \uparrow$ & $\mathrm{D}$ & $\mathrm{D}$ & $\leftrightarrow$ & Sujeto 19 & $\mathrm{B} / \mathrm{C}$ & $\mathrm{F}$ & $\uparrow \uparrow \uparrow$ & $\mathrm{B} / \mathrm{C}$ & $\mathrm{D}$ & $\uparrow$ \\
\hline Sujeto 7 & $\mathrm{B} / \mathrm{C}$ & $\mathrm{F}$ & $\uparrow \uparrow \uparrow$ & $\mathrm{A}$ & $\mathrm{E}$ & $\uparrow \uparrow \uparrow$ & Sujeto 20 & $\mathrm{D}$ & $\mathrm{D}$ & $\leftrightarrow$ & $\mathrm{A}$ & $\mathrm{D}$ & $\uparrow \uparrow \uparrow$ \\
\hline Sujeto 8 & $\mathrm{B} / \mathrm{C}$ & $\mathrm{D}$ & $\uparrow$ & $\mathrm{A}$ & $\mathrm{C}$ & $\uparrow$ & Sujeto 21 & $\mathrm{A}$ & $\mathrm{E}$ & $\uparrow \uparrow \uparrow$ & $\mathrm{A}$ & $\mathrm{D}$ & $\uparrow \uparrow$ \\
\hline Sujeto 9 & $\mathrm{A}$ & $\mathrm{D}$ & $\uparrow \uparrow$ & $\mathrm{D}$ & $\mathrm{E}$ & $\uparrow$ & Sujeto 22 & $\mathrm{A}$ & $\mathrm{E}$ & $\uparrow \uparrow \uparrow$ & $\mathrm{D}$ & $\mathrm{D}$ & $\leftrightarrow$ \\
\hline Sujeto 10 & $\mathrm{B} / \mathrm{C}$ & $\mathrm{F}$ & $\uparrow \uparrow \uparrow$ & $\mathrm{B} / \mathrm{C}$ & $\mathrm{D}$ & $\uparrow$ & Sujeto 23 & $\mathrm{B} / \mathrm{C}$ & $\mathrm{F}$ & $\uparrow \uparrow \uparrow$ & $\mathrm{B} / \mathrm{C}$ & $\mathrm{D}$ & $\uparrow$ \\
\hline Sujeto 11 & $\mathrm{A}$ & $\mathrm{E}$ & $\uparrow \uparrow \uparrow$ & $\mathrm{B} / \mathrm{C}$ & $\mathrm{E}$ & $\uparrow \uparrow$ & Sujeto 24 & $\mathrm{B} / \mathrm{C}$ & $\mathrm{D}$ & $\uparrow$ & $\mathrm{A}$ & $\mathrm{E}$ & $\uparrow \uparrow \uparrow$ \\
\hline Sujeto 12 & $\mathrm{B} / \mathrm{C}$ & $\mathrm{F}$ & $\uparrow \uparrow \uparrow$ & $\mathrm{B} / \mathrm{C}$ & $\mathrm{C}$ & $\uparrow$ & Sujeto 25 & $\mathrm{B} / \mathrm{C}$ & $\mathrm{F}$ & $\uparrow \uparrow \uparrow$ & $\mathrm{B} / \mathrm{C}$ & $\mathrm{D}$ & $\uparrow$ \\
\hline Sujeto 13 & $\mathrm{A}$ & $\mathrm{D}$ & $\uparrow \uparrow$ & $\mathrm{B} / \mathrm{C}$ & $\mathrm{E}$ & $\uparrow \uparrow$ & & & & & & & \\
\hline
\end{tabular}

\section{Evaluación del Ciclo de Mejora Docente}

\section{Aspectos de la experiencia que se pretenden incorporar a la práctica docente habitual.}

En general estoy muy satisfecho con la implementación del CIMA, ha superado mis expectativas y la experiencia ha sido realmente positiva, me he sentido muy cómodo en todo momento y aunque hubo momentos en que me pude sentir desbordado, al final la no se descontroló y el resultado alcanzado fue óptimo en términos generales.

Debido a la intrínseca complejidad de la estrategia seguida, era fundamental que el alumnado se comprometiera, por ello la idea de no obligar a nadie a participar, así como exigir un compromiso previo, fue fundamental para que fuese satisfactoria. Casi toda la clase se apuntó y la mayoría participó activamente.

Creo que proporcionarles una gran cantidad de materiales con los que pudiesen iniciar la investigación fue un acierto ya que la mayoría de los documentos de posición tuvieron una calidad bastante aceptable. De la misma manera, explicarles los procedimientos pormenorizadamente

Jornadas de Formación e Innovación Docente del Profesorado I № 2 (2019)

Esta obra se distribuye con la licencia Creative Commons

Reconocimiento-NoComercial-SinObraDerivada $\quad 4.0$ Internacional (CC BY-NC-ND 4.0.) 
ha sido decisivo para el correcto desarrollo de la experiencia.

Con objeto de lograr unos resultados mayores, para futuras ediciones debería no solo utilizar el formato en otras lecciones, sino otorgarle un rol preeminente en el proyecto docente, aumentando su peso en la calificación final de la asignatura.

\section{Principios didácticos que han guiado la experiencia y deben mantenerse}

- Es crucial elaborar una detallada secuencia de actividades antes de las sesiones, ya que esto nos dará seguridad a la hora de implementar el nuevo modelo didáctico y, al mismo tiempo, durante la elaboración de la secuencia somos capaces de identificar alternativas a las actividades finalmente seleccionadas. Esto es importante porque en el caso de que la realidad en el aula nos impida ceñirnos estrictamente a lo planeado, nos permitirá improvisar, pero apoyados en alternativas previamente estudiadas y trabajadas.

- Exigir compromiso al alumnado y hacerlo partícipe de un resultado final tangible, logra que se involucre de una manera más decidida en su proceso de enseñanza-aprendizaje.

- Con objeto de medir el progreso del alumnado es muy importante seguir manteniendo las escaleras de aprendizaje a través de cuestionarios iniciales y finales, ya que nos permite reorientar la elección de contenidos al nivel inicial que nos encontremos en el aula, y al mismo tiempo nos permite cuantificar el progreso que tiene lugar tras nuestro desempeño docente.

Jornadas de Formación e Innovación Docente del Profesorado | № 2 (2019)

Esta obra se distribuye con la licencia Creative Commons 


\section{Bibliografía}

Bain, K. (2005). Lo que hacen los mejores profesores universitarios. Valencia: Publicaciones Universidad de Valencia

Finkel, D. (2008). Dar clases con la boca cerrada. Valencia: Publicaciones Universidad de Valencia

Porlán, R. (Coord.). (2017). Enseñanza universitaria. Cómo mejorarla. Madrid: Morata

Jornadas de Formación e Innovación Docente del Profesorado | № 2 (2019)

Esta obra se distribuye con la licencia Creative Commons 